\title{
Respostas fisiológicas de recém-nascidos pré-termo submetidos ao Metódo Mãe-Canguru e a posição prona
}

\author{
Physical responses of pre-term newborn babies submitted \\ to the Kangaroo-Mother Care Method in Prone position
}

Maiara Dantas Olmedo', Giselle dos Santos Gabas', Leila Simone Foerster Merey², Ligia Stein de Souza', Karla de Toledo Candido Muller ${ }^{2}$, Mara Lisiane de Moraes dos Santos ${ }^{3}$, Cassia Fernandes Marques ${ }^{1}$

RESUMO I O Ministério da Saúde recomenda e incentiva a Atenção Humanizada ao recém-nascido de baixo peso utilizando-se o Método Mãe-Canguru (MMC) nas unidades integrantes do Sistema Único de Saúde (SUS). O objetivo deste estudo foi avaliar e comparar as respostas fisiológicas entre o MMC e a posição prona (PP), em recém-nascidos pré-termo (RNPT). Foi feito um estudo de intervenção, realizado entre setembro e outubro de 2009, composto por 20 RNPT, de ambos os sexos, com idade gestacional entre 24 a 36 semanas, estáveis hemodinamicamente, sendo classificados como grupo I (MMC) e grupo II (PP). Foram consideradas as variáveis: frequência cardíaca ( $F C$ ), frequência respiratória (FR), saturação periférica de oxigênio $\left(\mathrm{SatO}_{2}\right.$ ) e temperatura corporal $(\mathrm{T})$. As mensurações foram realizadas por três dias consecutivos, antes e 60 min após a aplicação das técnicas. No grupo PP, a FR aferida antes foi significativamente maior do que a aferida após a intervenção, nos $1^{\circ}$ e $3^{\circ}$ dias ( $p<0,0001$; $p<0,006$ ); enquanto que, no MMC, a FR apresentou diferença significativa somente no 30 dia $(p<0,006)$. A FC apresentou redução entre os momentos no 30 dia, nos grupos PP $(p<0,02)$ e no MMC $(p<0,04)$. No grupo $\mathrm{PP}$, a variável $\mathrm{SatO}_{2}$ apresentou significativo aumento nos $1^{\circ}(p<0,02)$ e 30 dias $(p<0,02)$, entre os momentos de coleta, e no 30 dia do MMC $(p<0,04)$. Não foram observadas alterações na FR, FC, T e SatO ${ }_{2}$ com a aplicação do MMC e PP, não havendo melhor desempenho
ABSTRACT I The Ministry of Health recommends and looks forward to the Humanized Attention of low weight newborn babies using the Kangaroo-Mother Care Method (MCM) in Unified Health System units. The aim of this work was to evaluate and compare the physiological responses between the MMC and the Prone Position (PP) in pre-term newborn babies (PNB). Intervention study, realized between September and October of 2009, was performed. It was formed by 20 PNB, both sexes, with gestational ages between 24 to 36 weeks, hemodynamic stable, classified as group I (MCM) and group II (PP). The following variables were considered: heart frequency (HF), breathing frequency (BF), periphery saturation of oxygen $\left(\mathrm{SatO}_{2}\right)$, and body temperature $(T)$. All measurements were realized for three consecutive days, before and 60 min after applying the procedures. On the PP group, the HF checked before was meaningfully higher than the one checked after the procedure, on the first and third days ( $p<0.0001 ; p<0.006)$. On the MCM, the HF presented meaningful difference only on the third day $(p<0.006)$. The HF presented meaningful reduction between the moments of the third day, on the groups PP $(p<0.02)$ and MCM $(p<0.04)$. On the PP group, the variable $\mathrm{SatO}_{2}$ presented meaningful raise on the first $(p<0.02)$ and third day $(p<0.02)$ between the moments of the data collects, and on the third day of the MCM $(p<0.04)$. No changes were observed on the BF, HF, T and SatO ${ }_{2}$ with the application of the MCM and PP, and there were no different results between the groups. We noticed decreasing

Estudo desenvolvido no Hospital Universitário da Universidade Federal de Mato Grosso do Sul (UFMS) - Campo Grande (MS), Brasil. 'Fisioterapeuta Graduada pela Universidade Católica Dom Bosco (UCDB) - Campo Grande (MS), Brasil.

2Mestre em Saúde e Desenvolvimento na Região Centro pela UFMS - Campo Grande (MS), Brasil; Docente da UCDB - Campo Grande (MS), Brasil.

${ }^{3}$ Doutora em Ciências da Saúde pelo convênio multi-institucional Rede Centro-Oeste Universidade de Brasília/Universidade Federal de Goiás/UFMS - Campo Grande (MS), Brasil. 
em relação aos grupos. Observamos diminuição da FR após a aplicação do MMC e PP em momentos isolados e aumento da SatO, no 30 dia após o MMC.

Descritores I prematuro; decúbito ventral; Método Mãe-Canguru. on the BF after application of the MMC and PP on occasional moments and increasing of $\mathrm{SatO}_{2}$ on the third day after the MMC. Keywords I infant, premature; prone position; Kangaroo-Mother Care Method.

\section{INTRODUÇÃO}

Os programas de intervenção precoce envolvendo recém-nascidos pré- termo têm produzido evidências substanciais que potencializam a interação da criança com o meio ambiente através de estímulos proprioceptivos, táteis, vestibulares, cinestésicos, auditivos e visuais. Os princípios dessas intervenções se baseiam em dar condições para que o neonato se auto-organize, induzindo-o a interagir com os pais, cuidadores e o meio, buscando respostas próximas ao padrão de normalidade e à inibição da aprendizagem de movimentos e posturas anormais $^{1,2}$.

$\mathrm{O}$ atendiento perinatal tem sido foco das atenções do Ministério da Saúde, já que nesse componente, juntamente com o pré-natal, reside o maior desafio para a redução da mortalidade infantil. Nesse sentido, o Ministério da Saúde, pela Portaria Gabinete do Ministro/Ministério da Saúde (GM/MS) No 072 de 02 março de 2000, recomenda e incentiva a Atenção Humanizada ao recém-nascido de baixo peso, por meio do Método Mãe-Canguru (MMC), em todas as unidades médico-assistenciais integrantes do Sistema Único de Saúde (SUS), propondo a aplicação do método em três etapas: unidades de terapia intensiva neonatal (UTIN), unidades intermediárias (UI) e acompanhamento ambulatorial $(\text { follow up })^{3-5}$.

A literatura consultada sugere que o MMC contribui para alterações benéficas nos sinais vitais dos RNPT promovendo melhora da temperatura corporal e aumento da saturação periférica de oxigênio, com consequente melhora na oxigenação tecidual e redução na frequência respiratória ${ }^{6-8}$.

O MMC é um método que merece grande incentivo, por ser simples e de baixo custo, podendo ser aplicado em qualquer hospital, trazendo importante contribuição para a fisioterapia, como um recurso adicional no tratamento de RNPT de baixo peso, mostrando-se eficaz na manutenção e melhora dos sinais vitais ${ }^{7}$.

Apesar dos benefícios descritos referentes à utilização do MMC, existem alguns complicadores para aplicação do método, dentre eles destacamos a não permanência dos pais durante o período de internação e recusa das mães quando decorre de uma gestação indesejada, daí a inquietação em utilizarmos outro posicionamento que possa favorecer o desenvolvimento neuropsicomotor. A posição prona (PP) pode ser vista como um posicionamento favorável nessas situações, não na tentativa de substituir o MMC, mas proporcionar um conforto similar, devido aos benefícios que essa posição pode acarretar no RNPT, como melhora dos índices de oxigenação e alterações referentes à mecânica respiratória ${ }^{9}$.

A proposta deste estudo foi avaliar e comparar as respostas fisiológicas entre o MMC e a PP em RNPT. Foram consideradas as variáveis: frequência cardíaca (FC), frequência respiratória (FR), saturação periférica de oxigênio $\left(\mathrm{SatO}_{2}\right)$ e temperatura corporal $(\mathrm{T})$.

\section{METODOLOGIA}

Tratou-se de um estudo de intervenção, descritivo, realizado na Unidade Intermediária do Hospital Universitário de Mato Grosso do Sul, com RNPT oriundos da maternidade do hospital, ou de outras localidades, no período de setembro a outubro de 2009. O estudo foi realizado após aprovação do Comitê de Ética em Pesquisa com Seres Humanos da Universidade Católica Dom Bosco.

Foram incluídos 20 RNPT, com idade gestacional entre 24 e 36 semanas avaliada pelo método Ballard ${ }^{10}$, com peso adequado ou não para a idade, independente da idade cronológica, nascidos na maternidade do Hospital Universitário (HU) que estivessem em respiração espontânea, com monitorização não invasiva, estáveis hemodinamicamente, ou seja, sinais vitais dentro da faixa de normalidade para a faixa etária por pelo menos $24 \mathrm{~h}$, oriundos da UTIN em atendimento na UI da Faculdade de Medicina Dr. Helio Mandetta da Universidade Federal de Mato Grosso do Sul, com tempo de internação $\leq 28$ dias. Esses foram randomizados em dois grupos através de sorteio aleatório, sendo o grupo I composto 
por aqueles submetidos ao MMC e o grupo II composto por aqueles que foram posicionados em PP.

Os critérios de não inclusão foram: recém-nascidos que apresentassem malformações congênitas, hipóxia perinatal, assim como aqueles que tivessem algum tipo de síndrome que comprometesse o seu desenvolvimento, que estivessem em ventilação mecânica invasiva ou não invasiva no momento da coleta, com instabilidade hemodinâmica e/ou piora clínica que impedisse a coleta dos dados.

Foram excluídos da pesquisa os RNPT que receberam alta antes do término da coleta e/ou aqueles que os pais optassem por não mais fazerem parte da pesquisa.

Após selecionados, as mães e bebês foram avaliados por três dias consecutivos, no período vespertino, respeitando a rotina hospitalar.

A avaliação dos bebês ocorreu após uma hora do término de cada mamada e procedeu-se com os mesmos na posição dorsal.

Ambos os grupos foram avaliados por três dias consecutivos, e submetidos à aferição das variáveis $\mathrm{FC}, \mathrm{FR}$, $\mathrm{SatO}_{2}$ e $\mathrm{T}$, no momento que antecedia a colocação do $\mathrm{RN}$ ao posicionamento (MMC ou PP), conforme o grupo a que pertencia, e imediatamente após os $60 \mathrm{~min}$ de posicionamento.

Durante a avaliação dos RNPT que foram posicionados no MMC, a mãe permaneceu sentada em uma poltrona, na posição Fowler com o tórax despido; em seguida, o recém-nascido foi colocado em posição vertical e fetal em seu peito em contato pele a pele, envolto em uma faixa de malha durante $60 \mathrm{~min}$.

$\mathrm{Na} \mathrm{PP}$, os neonatos foram posicionados somente de fraldas na incubadora aquecida, em posição fetal com uma sustentação toracoabdominal para maior estabilização da mecânica respiratória, durante $60 \mathrm{~min}$.

As alterações das respostas fisiológicas foram mensuradas por meio da observação das seguintes variáveis: FC em batimentos cardíacos por minuto (bpm) e $\mathrm{SatO}_{2}$ (em percentual de oxigênio) através do oxímetro de pulso da marca DIXTAL ${ }^{\circledR}$ modelo DX2515, posicionado no membro inferior direito (MID) conforme padronização, durante $3 \mathrm{~min}$. A T foi registrada em graus Celsius por meio de um termômetro clínico de coluna de mercúrio da marca Premium ${ }^{\circledR}$ posicionado na prega axilar do membro superior direito (MSD) em posição horizontal paralela ao eixo sagital por um período de 4 min e a FR em incursões respiratórias por minuto (irpm).

Apenas uma das pesquisadoras ficou responsável pela mensuração da variável FR durante a pesquisa, para garantirmos a fidedignidade da coleta.
Para a comparação dos resultados nos três dias de avaliação em cada um dos grupos, foi utilizado o teste ANOVA de uma via de medidas repetitivas. Para comparação dos resultados entre os grupos, foi utilizado o teste $t$ de Student para amostras não pareadas, já para comparar o antes e o após de cada método se fez necessário utilizar o teste $t$ de Student para amostras pareadas, ambos com nível de significância de 5\%. Os resultados das variáveis avaliadas neste estudo foram apresentados na forma de estatística descritiva e na forma de tabelas, sendo a análise estatística realizada utilizando-se o software SigmaStat ${ }^{11}$ versão 2.0.

\section{RESULTADOS}

Participaram da pesquisa 20 RNPT, sendo 12 do sexo feminino e 8 do sexo masculino, com idade gestacional variando entre 24 a 37 semanas, com média de idade em dias de 7,1 $\pm 1,8$ (média \pm erro padrão), sendo 10 RNPT alocados no grupo I MMC e 10 no grupo II PP através de sorteio aleatório.

Em relação às crianças do grupo $\mathrm{MMC}$, os dados vitais $\mathrm{FR}, \mathrm{FC}, \mathrm{T}$ e $\mathrm{SatO}_{2}$ não apresentaram diferenças entre os $1^{\circ}, 2^{\circ}$ e $3^{\circ}$ dias de tratamento, considerando para esse cálculo a média entre os valores aferidos antes e após a aplicação das referida técnicas ( $\mathrm{p}=0,98 ; \mathrm{p}=0,64$; $\mathrm{p}=0,23 ; \mathrm{p}=0,94$; respectivamente) (Tabela 1 ).

As crianças pertencentes ao grupo $\mathrm{PP}$ tiveram seus dados vitais $\mathrm{FR}, \mathrm{FC}, \mathrm{SatO}_{2}$ e T aferida também nos dois momentos e não foi significativa a diferença entre os valores médios obtidos nos 3 dias de observação ( $\mathrm{p}=0,46$; $\mathrm{p}=0,98 ; \mathrm{p}=0,07 ; \mathrm{p}=0,39$, respectivamente). Tais dados demonstraram não haver efeito acumulativo ao longo de 3 dias de terapia, tanto do MMC, como da PP (Tabela 1).

Não foi significativa a diferença entre os valores médios dos dados vitais FR, FC, $\mathrm{SatO}_{2}$ e T, observados nos $1^{\circ}, 2^{\circ}$ e $3^{\circ}$ dias de tratamento nos $\mathrm{RN}$ do grupo MMC comparados aos do grupo PP por meio do teste $t$ de Student não pareado. Sendo assim, fica evidente não haver melhor desempenho em um grupo em relação ao outro, independente do dia de terapia. Os valores médios observados e o erro padrão da média, em cada uma das variáveis analisadas estão representados na Tabela 1.

De forma pareada, por meio do teste $t$ de Student, foram comparados o valor médio dos dados vitais $\mathrm{FR}$, FC e SatO , coletados antes e após a aplicação das técnicas $\mathrm{MMC}$ e $\mathrm{PP}$, no $1^{\circ}$ e no $3^{\circ}$ dia de terapia, conforme apresentado na Tabela 2. 
Tabela 1. Média e erro padrão dos parâmetros fisiológicos dos 20 recém-nascidos pré-termo, nos três dias de aplicação do Método Mãe-Canguru e posição prona. Campo Grande (MS), 2009

\begin{tabular}{|c|c|c|c|}
\hline \multirow{2}{*}{ Momento da coleta } & \multicolumn{2}{|c|}{$\begin{array}{l}\text { Parâmetros vitais por tratamento fisioterápico } \\
\text { (média } \pm \text { erro padrão da média) }\end{array}$} & \multirow{2}{*}{ Valor $\mathrm{p}^{(\mathrm{b})}$} \\
\hline & $\begin{array}{l}\text { Método Mãe-Canguru } \\
\qquad(n=10)\end{array}$ & $\begin{array}{l}\text { Posição Prona } \\
(n=10)\end{array}$ & \\
\hline \multicolumn{4}{|c|}{ Frequência respiratória } \\
\hline 10 dia & $49,10 \pm 2,05$ & $55,55 \pm 2,5$ & 0,67 \\
\hline $20 \mathrm{dia}$ & $48,55 \pm 2,74$ & $51,90 \pm 1,5$ & 0,30 \\
\hline $3^{\circ}$ dia & $49,00 \pm 2,14$ & $48,30 \pm 1,8$ & 0,80 \\
\hline Valor $\mathrm{p}^{(\mathrm{a})}$ & 0,98 & 0,46 & \\
\hline \multicolumn{4}{|l|}{ Frequência cardíaca } \\
\hline 10 dia & $144,9 \pm 3,8$ & $132,6 \pm 4,6$ & 0,06 \\
\hline $2^{\circ} \mathrm{dia}$ & $143,3 \pm 4,7$ & $132,4 \pm 3,0$ & 0,07 \\
\hline 30 dia & $139,8 \pm 3,1$ & $135,9 \pm 4,5$ & 0,07 \\
\hline Valor $\mathrm{p}^{(\mathrm{a})}$ & 0,64 & 0,98 & \\
\hline \multicolumn{4}{|l|}{ Saturação de $\mathrm{O}_{2}$} \\
\hline $10 \mathrm{dia}$ & $93,7 \pm 0,7$ & $95,2 \pm 0,6$ & 0,12 \\
\hline $2^{\circ}$ dia & $94,7 \pm 0,6$ & $95,0 \pm 0,6$ & 0,73 \\
\hline 30 dia & $95,7 \pm 0,7$ & $96,7 \pm 0,4$ & 0,12 \\
\hline Valor $\mathrm{p}^{(\mathrm{a})}$ & 0,23 & 0,07 & \\
\hline \multicolumn{4}{|l|}{ Temperatura } \\
\hline 10 dia & $36,8 \pm 0,0$ & $36,9 \pm 0,2$ & 0,40 \\
\hline $2^{\circ}$ dia & $36,8 \pm 0,1$ & $36,7 \pm 0,1$ & 0,57 \\
\hline 30 dia & $36,7 \pm 0,1$ & $36,7 \pm 0,1$ & 0,21 \\
\hline Valor $\mathrm{p}^{(\mathrm{a})}$ & 0,94 & 0,39 & \\
\hline
\end{tabular}

(a) Teste ANOVA de vias repetidas; ${ }^{(b)}$ Teste $t$ de Student não pareado

Tabela 2. Média e erro padrão dos parâmetros fisiológicos dos 20 recém-nascidos pré-termo, nos 10 e 30 dias de aplicação do Método Mãe-Canguru e posição prona. Campo Grande (MS), 2009

\begin{tabular}{|c|c|c|c|c|c|}
\hline \multirow{3}{*}{ Variável } & \multirow{3}{*}{ Momento da coleta } & \multicolumn{4}{|c|}{$\begin{array}{l}\text { Parâmetros vitais por tratamento fisioterápico } \\
\text { (média } \pm \text { erro padrão da média) }\end{array}$} \\
\hline & & \multicolumn{2}{|c|}{ Método Mãe-Canguru (dias) } & \multicolumn{2}{|c|}{ Posição prona (dias) } \\
\hline & & 10 & 30 & 10 & 30 \\
\hline \multicolumn{6}{|c|}{ Frequência respiratória } \\
\hline & Antes & $50,1 \pm 2,4$ & $51,1 \pm 2,5$ & $53,4 \pm 2,4$ & $51,4 \pm 1,4$ \\
\hline & Depois & $48,1 \pm 1,8$ & $46,9 \pm 1,8$ & $47,7 \pm 2,9$ & $45,2 \pm 2.4$ \\
\hline Valor $p^{(c)}$ & & 0,15 & $<0,006^{*}$ & $<0,0001^{*}$ & $<0,006^{*}$ \\
\hline \multicolumn{6}{|c|}{ Frequência cardíaca } \\
\hline & Antes & $147,6 \pm 4,3$ & $142,5 \pm 3,3$ & $133,2 \pm 5,5$ & $137,8 \pm 4,8$ \\
\hline & Depois & $142,3 \pm 4,5$ & $137,1 \pm 3,4$ & $132, \mathrm{O} \pm 4,2$ & $134,0 \pm 4,2$ \\
\hline Valor $\mathrm{p}^{(c)}$ & & 0,23 & $<0, \mathrm{O} 4^{*}$ & 0,73 & $>0,02^{*}$ \\
\hline \multicolumn{6}{|c|}{ Saturação de $\mathrm{O}_{2}$} \\
\hline & Antes & $93,5 \pm 0,1$ & $94,7 \pm 0,9$ & $94,5 \pm 0,8$ & $94,5 \pm 0,8$ \\
\hline & Depois & $94,4 \pm 0,9$ & $96,1 \pm 0,6$ & $96,0 \pm 0,4$ & $96 \pm 0,4$ \\
\hline Valor $\mathrm{p}^{(\mathrm{c})}$ & & 0,52 & $<0,04^{*}$ & $<0,02^{*}$ & $<0,02^{*}$ \\
\hline
\end{tabular}

(c) Teste $t$ de Student pareado

*Valor estatisticamente significativo

Em relação à $\mathrm{PP}$, observou-se que a FR aferida antes foi significativamente maior do que a aferida após da aplicação da técnica, tanto no $1^{\circ}$ como no $3^{\circ}$ dia $(\mathrm{p}<0,0001 ; \mathrm{p}<0,006)$. No grupo MMC, a FR também apresentou diferença significativa entre esses momentos de coleta no $3^{\circ}$ dia $(p<0,006)$, mas a diferença não foi significativa no $1^{\circ}$ dia terapêutico $(\mathrm{p}=0,15)$.
A comparação entre os valores médios de FC, coletados antes e após a terapia, apresentaram uma significativa redução no $3^{\circ}$ dia, considerando-se tanto a aplicação da técnica MMC, como da $\mathrm{PP}(\mathrm{p}<0,04 ; \mathrm{p}<0,02$; respectivamente). Entretanto, tal redução não foi significativa no $1^{\circ}$ dia quando comparados tais momentos, fato observado no método MMC ( $\mathrm{p}=0,23)$ assim como na $\mathrm{PP}(\mathrm{p}=0,73)$. 
No grupo MMC, o aumento de $\mathrm{SatO}_{2}$ ocorreu apenas no $3^{\circ}$ dia $(\mathrm{p}<0,04)$, sendo que no $1^{\circ}$ dia esse aumento não foi significativo $(\mathrm{p}=0,52)$. No grupo $\mathrm{PP}$, a variável $\mathrm{SatO}_{2}$ apresentou significativo aumento no $1^{\circ}$ dia, assim como no $3^{\circ}$ dia, entre os momentos antes e depois da intervenção $(p<0,02 ; p<0,02$, respectivamente).

\section{DISCUSSÃO}

Este estudo teve como objetivo avaliar e comparar as respostas fisiológicas em RNPT, submetidos à aplicação do MMC e da PP. Em relação à variável FR, observamos uma diminuição após a aplicação de cada método, o que nos leva a pesar que esse achado se deva às condições anatômicas e fisiológicas dos recém-nascidos, comum nessa faixa etária, a qual predispõe a ocorrência de respirações periódicas, crises de apneias e distorções de caixa torácica.

O MMC pode ter proporcionado maior estabilização aos parâmetros fisiológicos destes RNPT devido contato pele a pele com a mãe e maior vínculo materno, assim como na PP ocorreu melhora da mecânica pulmonar e diminuição das assincronias toracoabdominais quando selecionado esse posicionamento ${ }^{12}$. Esse achado vem de encontro com os resultados encontrados por Azevedo et al. ${ }^{13}$, que avaliaram os estados comportamentais de 44 recém-nascidos com peso inferior a $1.500 \mathrm{~g}$, em ventilação mecânica, antes, durante e após o MMC e observaram que o MMC favoreceu o sono, principalmente o profundo e que $52,3 \%$ dos $\mathrm{RN}$ permaneceram nesse estado, comparado aos períodos antes $(6,8 \%)$ e após $(13,6 \%)$. Maia et al. ${ }^{5}$, em seu estudo, recomendam a MMC como sendo uma medida de escolha no manejo da dor de RNPT, por ser de baixo custo e não farmacológico.

Resultados semelhantes foram encontrados por Lundington-Hoe et al. ${ }^{14}$, em um estudo randomizado envolvendo 28 RNPT com idade gestacional inferior a 32 semanas, onde foi observado, por meio de eletroencefalograma, que o grupo submetido ao MMC por até $3 \mathrm{~h}$ apresentou menor agitação durante todo o período do estudo, quando comparado ao grupo que recebeu os cuidados na incubadora. Do mesmo modo, no estudo de Bauer et $\mathrm{al} .{ }^{15}$, onde foram avaliados 27 RNPT, entre 25-30 semanas, respirando espontaneamente, observou-se maior duração de sono durante o MMC, quando comparado ao grupo controle: $90 \%$ do tempo para os de idade gestacional entre $25-27$ semanas e $97 \%$ para os de idade gestacional entre 28-30 semanas.
O sono profundo é considerado um momento favorável ao desenvolvimento, inclusive cerebral, pois nesse estado há maior conservação de energia, especialmente para os RNPT ${ }^{16,17}$. De acordo com a recomendação para implementação do MMC em bebês pré-termo a partir de 30 semanas de idade gestacional, desenvolvido por Ludinton-Hoe et al. ${ }^{18}$, o MMC pode ser utilizado como estratégia para o favorecimento do estado de sono ${ }^{19}$.

Lioy e Manginello ${ }^{20}$ observaram 10 RNPT submetidos à $\mathrm{PP}$ e descreveram que houve uma diminuição da FR em bebês com síndrome do desconforto respiratório. Apesar da diferença entre os trabalhos citados, relacionada à condição clínica dos envolvidos, observamos uma concordância em relação à diminuição dessa variável, o que nos leva a pensar que o MMC pode exercer influência na FR independente da homogeneidade da amostra.

Em relação à $\mathrm{FC}$, observamos uma diminuição quando comparado o antes e após a aplicação dos métodos. Almeida et al. ${ }^{6}$ avaliaram 22 RNPT sem doenças associadas, e observaram uma tendência à diminuição da FC, devido a ausência de estresse físico e psicológico do bebê enquanto este permanecia junto ao seio materno no MMC. Mendonza et al. ${ }^{21}$, relataram, em seu estudo, que a FC estabilizou enquanto os RNPT eram posicionados em PP. Acreditamos que a diminuição dessa variável, assim como a diminuição da FR relatada acima, possam ter ocorrido devido ao período de sono profundo comum na população estudada, sendo que $80 \%$ do seu dia eles permanecem em sono rapid eye movement (REM - movimento rápido dos olhos), período em que podemos observar mudanças comportamentais importantes.

Em relação a variável $\mathrm{SatO}_{2}$, não observamos uma diferença estatística significativa nos 3 dias de avaliação; contudo, quando analisamos os valores da $\mathrm{SatO}_{2}$ nos momentos antes e após a realização dos métodos, os resultados foram estatisticamente significativos, conforme ilustrado na Tabela 2. Isso revela que os resultados obtidos com os métodos não oferecem efeito em longo prazo, concordando com Almeida et al. ${ }^{6}$, que descreveram sobre a eficácia do MMC nos sinais vitais de RNPT de baixo peso, relatando terem observado um aumento da oxigenação tecidual, evidenciada pelo aumento da $\mathrm{SatO}_{2}$, após a aplicação do método. Isso pode ser explicado devido ao estado de relaxamento do bebê que provavelmente diminui o consumo de oxigênio e melhora a oxigenação tecidual.

Paiva et al. ${ }^{22}$ relataram que a PP também proporciona uma melhora significativa na oxigenação dos RNPT, 
pois reduzem os fatores que contribuem para o colabamento alveolar predispondo a uma redistribuição da ventilação e perfusão.

A PP garante um melhor sincronismo toracoabdominal, por meio da estabilização das costelas, melhor incursão diafragmática garantindo maior eficácia da mecânica respiratória, além de determinar a descompressão e reexpansão alveolar dos seguimentos dorsais, que são áreas de maior atelectasia na posição supina, podendo, ainda, ocorrer o deslocamento do coração para região ventral havendo uma compressão direcionada ao esterno, resultando em uma pressão transpulmonar suficiente para exceder a pressão de abertura das vias aéreas nas regiões dorsais dos pulmões, aumentando assim o volume disponível para ventilação ${ }^{23,24}$.

Assim como a FR, FC e $\mathrm{SatO}_{2}$ são importantes para garantir uma condição hemodinâmica favorável, a $\mathrm{T}$ do RNPT também exerce um papel fundamental no equilíbrio homeostático. O MMC, devido ao contato pele a pele entre mãe e filho, evita a perda de calor corporal, favorecendo a homeostasia, que é regulada pelo balanço entre a termogênese e a termólise, além de estar associada à manutenção ou aumento de calor durante essa prática ${ }^{25}$.

Em nossa pesquisa não observamos significativa diferença quanto a temperatura corporal entre os grupos, discordando dos estudos de Basseto ${ }^{25}$ e Miltersteiner \& Miltersteiner ${ }^{1}$, que analisaram a temperatura corporal durante a aplicação do MMC e encontraram um aumento significativo na T do RNPT, levando a acreditar que isso tenha ocorrido pela utilização do MMC que leva a uma melhora do controle térmico e menor perda de calor. Entretanto, Blaymore et al. ${ }^{16}$ verificaram que a estabilidade da temperatura axilar e retal em RNPT puderam ser observadas 10 min após a colocação desses em posição MMC.

Não encontramos na literatura consultada estudos indicando aumento da temperatura corporal em RNPT submetidos à $\mathrm{PP}$, compatível com o resultado encontrado em nosso estudo. Diante desse achado, acreditamos que a estabilização da temperatura deve-se à ausência de deslocamento do RN da incubadora, evitando o estresse físico e psicológico, que leva a um aumento do seu metabolismo e consumo de oxigênio com consequente diminuição da homeostasia térmica.

Consideram-se como limitação deste estudo o tempo de avaliação dos RNPT apenas durante três dias consecutivos, impossibilitando uma análise profunda na tentativa de verificar o efeito dos métodos em longo prazo. Estudos futuros são necessários, uma vez que os resultados deste trabalho não permitem generalizações, já que investigações em populações muito vulneráveis necessitam de avaliações com o maior rigor metodológico possível.

Ao final dos três dias de tratamento, não foram observadas alterações na FR, FC, $\mathrm{T}$ e $\mathrm{SatO}_{2}$, em nenhum dos grupos estudados, em relação aos dados iniciais, e nem quando comparadas as variáveis entre os grupos. Quando comparadas as variáveis medidas antes e após 60 min da aplicação da PP ou MMC, nos $1^{\circ}$ e $3^{\circ}$ dias de tratamento, constatou-se redução da $\mathrm{FR}$ e aumento da $\mathrm{SatO}_{2}$ no grupo $\mathrm{PP}$, nos $1^{\circ}$ e $3^{\circ}$ dias de tratamento, e no grupo MMC apenas no $3^{\circ}$ dia; além de redução da $\mathrm{FC}$ no $3^{\circ}$ dia, em ambos os grupos.

Concluindo-se, assim, que as técnicas aplicadas foram efetivas na melhora da $\mathrm{FR}, \mathrm{SatO}_{2}$ e $\mathrm{FC}$ pelo menos até 60 min após a aplicação das mesmas, sendo mais evidentes com a PP, mas tais resultados não foram observados quando relacionados os dados do primeiro e terceiro dias de tratamento.

\section{REFERÊNCIAS}

1. Miltersteiner AR, Miltersteiner DR. Respostas fisiológicas da Posição Mãe-Canguru em bebês pré-termos de baixo peso e ventilando espontaneamente. Rev Bras Saúde Materno-Infant. 2003;3(4):23-32.

2. Carvalho MR. Método mãe canguru de atenção ao prematuro. Rio de Janeiro: BNDES; 2011.

3. Brasil. Ministério da Saúde. Atenção humanizada ao recém-nascido de Baixo Peso - Método Mãe-Canguru - Brasília: Manual Técnico; 2002.

4. Gontijo TL, Meireles AL, Malta DC, Proietti, FA, Xavier CC. Avaliação da implantação do cuidado humanizado aos recém-nascidos com baixo peso - método canguru. J Pediatr. 2010;86(1):33-9.

5. Maia FA, Azevedo VMGO, Gontijo FO. Os efeitos da posição canguru em resposta aos procedimentos dolorosos em recém-nascidos pré-termo: uma revisão da literatura. Rev Bras Ter Intensiva. 2011;23(3):370-3

6. Almeida CM, Almeida AFN, Forti EMP. Efeitos do Método Mãe Canguru nos sinais vitais de recém nascidos pré-termo de baixo peso. Rev Bras Fisioter. 2007:11(1):1-5.

7. Dood VL. Implications of kangaroo care for growth and development in preterm infants. J Obstet Gynecol Neonatal Nurse. 2005;34(2):218-32.

8. Bohnhorst B, Heyne T, Peter CS, Poets CF. Skin-to-skin (kangaroo) care, respiratory control, and thermoregulation. J Pediatr. 2001;138(2):193-7.

9. Wagaman MJ, Shutack JG, Moomjian AS, Schwartz JG, Shaffer TH, Fox WW. Improved oxygenation and lung compliance with prone positioning of neonates. J Pediatr. 1979;94(5):787-91.

10. Ballard JL, Khoury JC, Wedig K, Wang L, Eilers-Walsman BL, Cip R. New Ballard score, expanded to include extremely premature infants. J Pediatr. 1991;19(3):417-23.

11. Shott, S. Statistics for health professionals. London: W.B. Saunders Company; 1990. 
12. Wolfson MR, Greenspan JS, Deras KS, Allen JL, Shaffer TIl. Effect of position on the mechanical interaction between the rib age and abdomem in preterm infants. J Appl Physiol. 1992;72(3):1032-8.

13. Azevedo VMGO, David RB, Xavier CC. Cuidado mãe canguru em recém-nascidos pré-termo sob suporte ventilatório: avaliação dos estados comportamentais. Rev Bras Saúde Matern Infant (Recife). 2011:11(2):133-8.

14. Lundington-Hoe SM, Johnson MW, Morgan K, Lewis T, Gutman J, Wilson PD, et al. Neurophysiologic assessment of neonatal sleep organization: preliminary results of a randomized, controlled trial of skin contact with preterm infants. Pediatrics. 2006;117(5): 909-23.

15. Bauer K, Pyper A, Sperling P, Uhrig C, Versmold H. Effects of gestational and postnatal age on body temperature, oxygen consumption, and activity during early skin-to-skin contact between preterm infants of 25-30-week gestation and their mothers. Pediatr Res. 1998;44(2):247-51.

16. Blaymore BJA, Ferguson AE, Morales Y, Liebling JAMS, Archer D, Oh W, et al. Comparison of skin-to-skin contact with standard contact in low birth weight infants who are breast-fed. Arch Pediatr Adolesc Med. 1996;150(12):1265-9.

17. Lima G, Quintero-Romero S, Cattaneo A. Feasibility, acceptability and cost of Kangaroo mother care in Recife, Brazil. Ann Trop Paediatr. 2000;20(1):22-6.
18. Ludinton-Hoe SM, Morgan K, Abouelfettoh A. A clinical guideline for implementation of kangaroo care with premature infants of 30 or more weeks postmenstrual age. Adv Neonatal Care. 2008;8(3):S3-S23.

19. Almeida H, Venancio SI, Sanches MTC, Onukis D. Impacto do método canguru nas taxas de aleitamento materno exclusivo em recémnascidos de baixo peso. J Pediatr (Rio J). 2010;86(3):250-3.

20. Lioy J, Manginello FP. Comparison of prone and supine positioning in the immediate postextubation period of neonates. J Pediatr. 1988;112(6):982-4.

21. Mendonza JC, Roberts $J \mathrm{~L}$, Cook LN. Postural effects on pulmonary function and heart rate of preterm infants with lung disease. J Pediatr. 1991;118(3):445-8.

22. Paiva KCA, Beppu OS. Posição prona. J Bras Pneumol. 2005:31(4):332-40

23. Rugolo LMSS. Assistência ao recém-nascido de muito baixo peso. In: UNESP. Departamento de Pediatria da Faculdade de Medicina de Botucatu. Condutas em pediatria. 4a ed. Rio de Janeiro: EPUB; 1999. p. 146-9.

24. Selestrin CC, Oliveira AG, Ferreira C, Siqueira AAF, Abreu LC, Murad N. Avaliação dos parâmetros fisiológicos em recém-nascidos prétermo em ventilação mecânica após procedimentos de fisioterapia neonatal. Rev Bras Cresc Desenvolv Hum. 2007:17(1):146-55.

25. Basseto MCA. Neonatologia - um convite à atuação fonoaudiológica. Rio de Janeiro: Lovise; 1998. 